\title{
Two-color fluorescence detection of Poly (ADP-Ribose) Polymerase-1 (PARP-1) cleavage and DNA strand breaks in etoposide-induced apoptotic cells
}

\author{
C. Soldani ${ }^{1}$, M.G. Bottone ${ }^{1}$, C. Pellicciari ${ }^{1}$, and A.I. Scovassi ${ }^{2}$
}

'Dipartimento di Biologia Animale and Centro di Studio per l'Istochimica del CNR, University of Pavia, Piazza Botta 10, and ${ }^{2}$ Istituto di Genetica Biochimica ed Evoluzionistica del CNR, Via Abbiategrasso 207, I-27100 Pavia, Italy.

Key words: apoptosis, etoposide, fluorescence, immunocytochemistry, PARP-1 cleavage, TUNEL

\section{SUMMARY}

During apoptosis, the nuclear enzyme Poly(ADPRibose) Polymerase-1 (PARP-1) catalyzes the rapid and transient synthesis of poly(ADP-ribose) from $\mathrm{NAD}^{+}$and becomes inactive when cleaved by caspases. The regulation of these two opposite roles of PARP-1 is still unknown. We have recently investigated PARP-1 activation/degradation in Hep-2 cells driven to apoptosis by actinomycin $\mathrm{D}$. In the present work, we have extended our analysis to the effect of the DNA damaging agent etoposide, and paid attention to the relationship between PARP-1 cleavage and DNA fragmentation. An original fluorescent procedure was developed to simultaneously identify in situ the $\mathrm{p} 89$ proteolytic fragment of PARP-1 (by immunolabeling) and DNA degradation (by the TUNEL assay). The presence of p89 was observed both in cells with advanced signs of apoptosis (where the PARP-1 fragment is extruded from the nucleus into the cytoplasm) and in TUNEL-negative cells, with only incipient signs of chromatin condensation; this evidence indicates that PARP-1 degradation in etoposide-treated apoptotic cells may precede DNA cleavage.

\section{INTRODUCTION}

Poly(ADP-ribosylation) is a post-translational modification involved in DNA damage signaling and DNA repair (for a review, see Bürkle, 2001). The biochemical reaction leading to poly(ADPribosylation) consists in the synthesis of ADP-ribose from $\mathrm{NAD}^{+}$, followed by the formation of polymers of different length and structure. It is generally accepted that this process also takes place during apoptosis, when the nuclear enzyme Poly(ADPRibose) Polymerase-1 (PARP-1) synthesizes poly(ADP-ribose); PARP-1 is also a target for caspases which cleave it into two fragments of $89 \mathrm{kDa}$ and 24 $\mathrm{kDa}$ (for a review, see Scovassi et al., 1998; Scovassi and Poirier, 1999). The timing of PARP-1 cleavage is still controversial, especially in relation to the poly(ADP-ribose) synthesis and to the occurrence of other apoptotic events.

To investigate in situ the accumulation of poly(ADP-ribose) as well as the presence of the PARP-1 p89 proteolytic fragment, we recently applied to human Hep-2 cells a treatment with actinomycin D (AMD), which induces apoptosis without DNA damage (Soldani et al., 2001). In cells exhibiting early apoptotic features, the polymer and p89 were found to be concomitantly present in the nucleus. Interestingly enough, we demonstrated that polymer synthesis is only carried out in relatively early apoptotic steps, whereas p89 is also detectable in late karyorrhexic cells. Moreover, we also observed that, during apoptosis, the immunopositivity for p89 moves from the nucleus into the cytoplasm (Soldani et al., 2001). 
As for the effect of DNA-damaging agents, we have previously reported that, in etoposide and bleomycin-treated HeLa and HL60 cells, a massive poly(ADP-ribose) accumulation takes place concomitantly with the occurrence of DNA laddering; the synthesis of poly(ADP-ribose) is also detectable, although to a lesser extent, in cells with only incipient signs of apoptosis (Negri et al., 1997; Donzelli et al., 1997). Western blot analyses confirmed the occurrence of PARP-1 cleavage in late apoptosis, when DNA degradation is massive (Scovassi et al., 1998). So far, however, the timing of PARP-1 cleavage in relation with incipient DNA degradation was never investigated on single cells.

In the present work, we aimed at further studying the precise sequence of these events in cells driven to apoptosis by the topoisomerase II inhibitor, etoposide. To this purpose, we developed a twocolor fluorescence procedure based on the immunofluorescent detection of $p 89$ fragment of PARP-1 and the TUNEL assay. The final counterstaining of DNA with a specific fluorochrome also allowed to visualize chromatin texture.

\section{MATERIALS AND METHODS}

Hep-2 cells grown on glass coverslips in Petri dishes were treated, in their esponential growth phase, with $10 \mu \mathrm{M}$ etoposide for $15 \mathrm{hr}$ and fixed with $70 \%$ ethanol for $30 \mathrm{~min}$. For the TUNEL procedure, cells were incubated for $1 \mathrm{hr}$ at $37^{\circ} \mathrm{C}$ with $50 \mu 1$ of the TUNEL mixture according to the manufacturer's instructions (Boehringer Mannheim, Germany). After washings with PBS containing $0.05 \%$ Tween-20 and $0.1 \%$ bovine serum albumin, cells were incubated for $1 \mathrm{hr}$ at room temperature (r.t.) with an anti-p89 polyclonal antibody (Cell Signaling Technology, Celbio s.r.l., Italy) diluted 1:100 in PBS, and then for $1 \mathrm{hr}$ at r.t. with an Alexa594-conjugated anti-rabbit IgG antibody (Molecular Probes, The Netherlands) diluted 1:200 in PBS. Dual-labeled cells were finally counterstained for DNA with Hoechst $33258(0.1 \mu \mathrm{g} / \mathrm{ml}$ in PBS for $10 \mathrm{~min}$ ), washed with PBS and mounted up-side-down on a non-fluorescent glass slide in a drop of Mowiol (Calbiochem, Inalco S.p.A., Italy). Micrographs were taken with an Olympus Camedia C-2000 Z mounted on an Olympus BX50 fluorescence microscope.

\section{RESULTS AND DISCUSSION}

As shown in Fig. 1, the presence of p89 (red fluorescence: c, g) was detectable in TUNEL-positive Hep-2 cells (green fluorescence: b, f), i.e. in cells with apoptotic DNA fragmentation and chromatin condensation (a, e). However, we also found PARP-1 degradation in cells which were not stained by the TUNEL assay (c, arrow) and did not show apparent signs of chromatin condensation or margination. Remarkably, in late apoptotic, karyorrhexic cells, p89 migrates from the nucleus into the cytoplasm $(\mathrm{g}, \mathrm{h})$.

The cleavage of PARP-1 and its relationship with apoptotic DNA fragmentation have already been investigated by fluorescence microscopy and flow cytometry in single HL60 cells treated with camptothecin ( $\mathrm{Li}$ and Darzynkiewicz, 2000). This is, however, the first attempt to simultaneously detect these two processes in situ, on the same cell sample. Our fluorescence approach also made it possible to demonstrate that PARP-1 degradation occurred in some etoposide-treated cells which were still negative for the TUNEL assay.

The migration of $\mathrm{p} 89$ from the nucleus into the cytoplasm (we already observed after AMD treatment: Soldani et al., 2001) was here confirmed as a possibly general feature of late apoptosis, irrespective of the inducing stimulus.

The immunopositivity for p89 even in late apoptotic steps suggests that this peptide is resistant to extensive protease degradation, as much as it occurs for other nuclear and nucleolar proteins (Biggiogera et al., 1997a,b; Zweier et al., 1997; Pellicciari et al., 1999). It is still to be elucidated whether p89 may also become part of the ectopic heterogeneous clusters of nuclear molecules which have been widely described in apoptotic cells (Biggiogera et al., 1997b, 1998; Pellicciari et al., 2000; Martelli et al., 2001).

It is worth recalling that the clearance of apoptotic bodies is essential for a safe apoptotic process to take place (for a review, see Dini, 2000); actually, in the presence of defective clearance mechanisms, late apoptotic events entailing the generation of novel molecular aggregates -such as those described above- could trigger the production of autoantibodies against nuclear components (Rosen and Casciola-Rosen, 1999; Rodenburg et al., 2000; Rovere et al., 2000). 

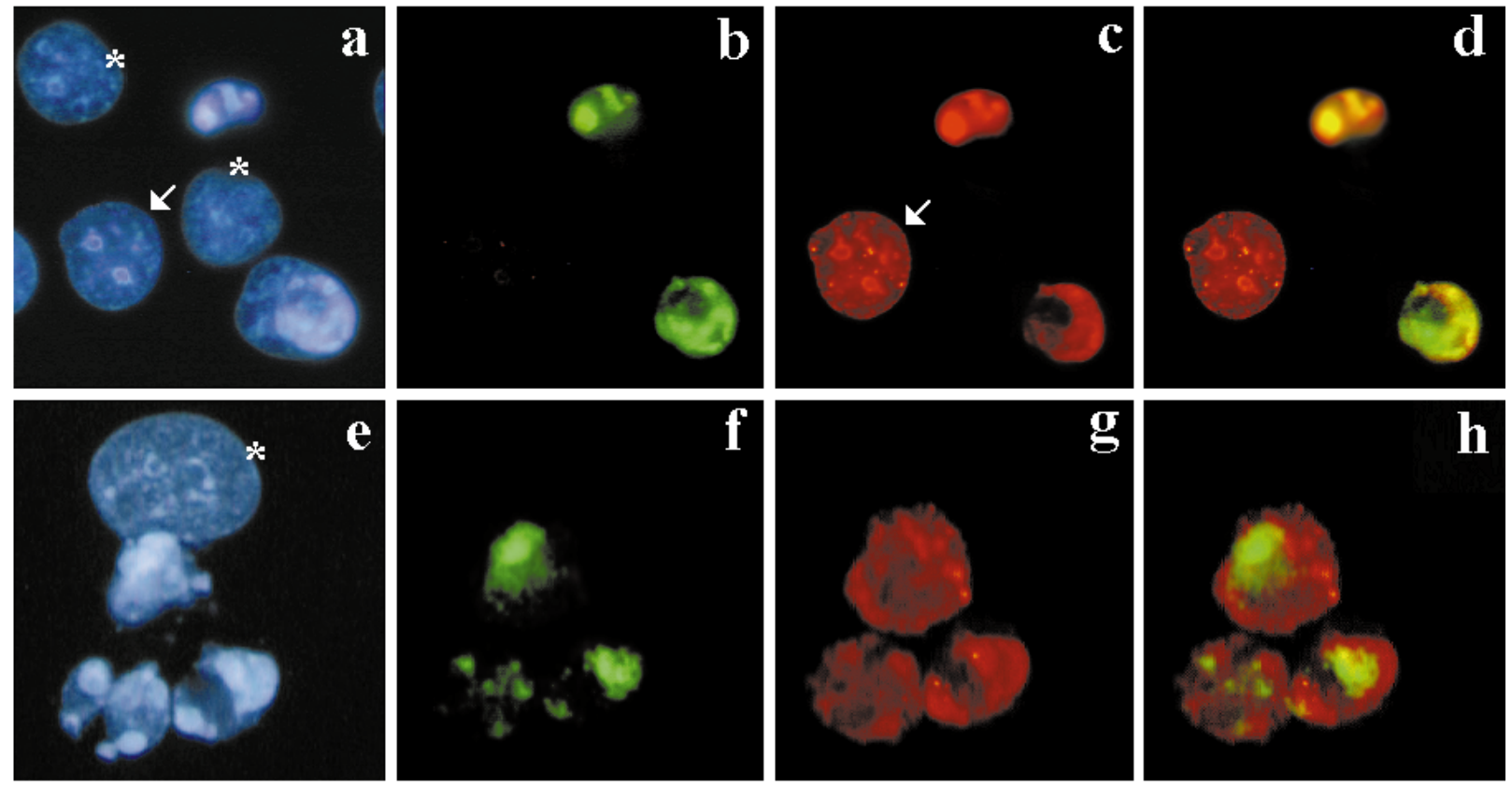

Fig. 1 - Detection of PARP-1 proteolysis and DNA fragmentation in etoposide-treated Hep-2 cells. a, e): Hoechst staining; b, f): TUNEL assay (green fluorescence); $\mathbf{c}, \mathbf{g}$ ): antibody against the $\mathrm{p} 89$ fragment of PARP-1 (red fluorescence); $\mathbf{d}, \mathbf{h})$ : merged images of green and red fluorescence, where the resulting yellow signals indicate colocalization of p89 and DNA degradation (in panel h, note that in late apoptotic cells with extensive karyorrhexis the red fluorescence for $\mathrm{p} 89$ is strictly cytoplasmic and cannot colocalize with the TUNEL green fluorescence). The arrow in a) and c) points to a TUNEL-negative cell with a positive signal for p89. Asteriscs indicate non apoptotic cells with non condensed chromatin which were never positive for either p 89 or the TUNEL assay.

\section{ACKNOWLEDGEMENTS}

C.S. is a student of the Dottorato di Ricerca in Biologia Cellulare. This work was supported by the University of Pavia (Fondo di Ateneo per la Ricerca, 2000 and Progetto Giovani Ricercatori). Thanks are due to Mrs. P. Veneroni for the excellent technical assistance.

\section{REFERENCES}

Biggiogera M., Bottone M.G., and Pellicciari C.: Nuclear ribonucleoprotein-containing structures undergo severe rearrangements during spontaneous thymocyte apoptosis. A morphological study at electron microscopy. Histochem. Cell Biol. 107, 331-336, 1997a.

Biggiogera M., Bottone M.G., Martin T.E., Uchiumi T., and Pellicciari C.: Still immunodetectable nuclear RNPs are extruded from the cytoplasm of spontaneously apoptotic thymocytes. Exp. Cell Res. 234, 512-520. 1997b.

Bürkle A.: Physiology and pathophysiology of poly(ADPribosylation). BioEssays 23, 795-806, 2001

Dini L.: Recognizing death: liver phagocytosis of apoptotic cells. Eur. J. Histochem. 44, 217-227, 2000.
Donzelli M., Negri C., Mandarino A., Rossi L., Prosperi E., Frouin I., Bernardi R., Bürkle A., and Scovassi A.I. Poly(ADP-ribose) synthesis: a useful parameter to identify apoptotic cells. Histochem. J. 29, 831-837, 1997.

Li X., and Darzynkiewicz Z.: Cleavage of poly(ADP-ribose) polymerase measured in situ in individual cells: relationship to DNA fragmentation and cell cycle position during apoptosis. Exp. Cell Res., 255, 125-132, 2000.

Martelli A.M., Zweyer M., Ochs R.L., Tazzari P.L., Tabellini G., Narducci P., and Bortul R.: Nuclear apoptotic changes: An overview. J. Cell Biochem. 82, 634-646, 2001.

Negri C., Donzelli M., Bernardi R., Rossi L., Bürkle A., and Scovassi A.I.: Multiparametric staining to identify apoptotic human cells. Exp. Cell Res. 234, 174-177, 1997.

Pellicciari C., Bottone M.G., Scovassi A.I., Martin T.E., and Biggiogera M.: Rearrangement of nuclear ribonucleoproteins and extrusion of nucleolus-like bodies during apoptosis induced by hypertonic stress. Eur. J. Histochem. 44, 247-254, 2000.

Pellicciari C., Bottone M.G., and Biggiogera M.: Restructuring and extrusion of nuclear ribonucleoproteins (RNPs) during apoptosis. Gen. Physiol. Biophys. 18, 50-52, 1999.

Rodenburg R.J.T., Raats J.M.H., Prujin G.J.M., and van Venrooij W.J.: Cell death: a trigger of autoimmunity? BioEssays 22, 627-636, 2000. 
Rosen A., and Casciola-Rosen L.: Autoantigens as substrates for apoptotic proteases: implications for the pathogenesis of systemic autoimmune disease. Cell Death Differ. 6, 6-12, 1999.

Rovere P., Fazzini F., Sabbadini M.G., and Manfredi A.A.: Apoptosis and systemic autoimmunity: the dendritic cell connection. Eur J Histochem. 44, 229-236, 2000.

Scovassi A.I., and Poirier G.G.: Poly(ADP-ribosylation) and apoptosis. Mol. Cell. Biochem. 199, 125-137, 1999.

Scovassi A.I., Denegri M., Donzelli M., Rossi L., Bernardi R., Mandarino A., Frouin I., and Negri C.: Poly(ADP-ribose) syn- thesis in cells undergoing apoptosis: an attempt to face death before PARP degradation. Eur. J. Histochem. 4, 251-258, 1998.

Soldani C., Lazzè M.C., Bottone M.G., Tognon G., Biggiogera M., Pellicciari C. and Scovassi A.I.: Poly(ADPribose) polymerase cleavage during apoptosis: when and where? Exp. Cell Res. 269, 193-201, 2001.

Zweier M., Riederer B.M., Ochs R.L., Fackelmayer F.O., Kohwi-Shigematsu T., Bareggi R., Narducci P., and Martelli A.M.: Association of nuclear matrix proteins with granular and threated nuclear bodies in cell lines undergoing apoptosis. Exp. Cell Res. 230, 325-366, 1997. 